\title{
Title: Regulation of lipid metabolism by microRNAs (miRNA)
}

\section{Editorial Comment}

Rothwelle Tate, Dino Rotondo \& Jillian Davidson Strathclyde Institute of Pharmacy and Biomedical Sciences, University of Strathclyde, Glasgow G4 ORE, Scotland, U.K.

Correspondence to D. Rotondo. Tel: +44 141548 3629;

fax: +44 141552 2562; e-mail: d.rotondo@strath.ac.uk

MicroRNAs (miRNAs) are a class of small non-coding RNAs, typically 22 nucleotides in length, which post-transcriptionally regulate gene expression. Mature miRNAs bind complementary sequences in the $3^{\prime}$ untranslated region (3' UTR) of target mRNAs and repress protein expression via a number of mechanisms including mRNA destabilisation and/or inhibition of translation [1]. So far, almost 36,000 mature miRNAs from 223 species have been classified with 2588 of them human (miRBase, Release 21) [2, 3-]. As a single miRNA is able to target hundreds of transcripts in humans, they are likely to influence regulation of all cellular events $[4,5]$ and their dysregulation has been correlated with disease pathogenesis [6]. In addition to acting endogenously, miRNAs can be released from cells where they may be involved in cell-cell communication and extracellular regulatory mechanisms and have been detected in many body fluids $[7,8]$. These extracellular, circulating miRNAs may be non-invasive biomarkers of disease and targets for therapeutic intervention $[9,10]$.

Individual miRNAs have been found to regulate specific genes involved in lipid metabolism. He et al. [11] ${ }^{-}$utilised target prediction to identify the possible regulation of lipoprotein lipase (LPL) by miR-590. They used synthetic miR-590 miRNA with human THP-1 macrophages to demonstrate for the first time that miR590 may be involved in atherosclerosis by negatively regulating lipid accumulation, lipid-laden foam cell formation, and pro-inflammatory cytokine (including TNF- $\alpha$ and IL-1 $\beta$ ) production via downregulation of LPL expression. In a related manner, it has been shown by Vickers et al. [12]- that a single microRNA, miR-223, plays a critical role co-ordinating lipid metabolism by post-transcriptionally regulating multiple genes which control uptake, efflux, and biosynthesis of cholesterol. miR223 appears to achieve this through the downregulation of the expression of both Hmgcs1 (3-hydroxy-3-methylglutaryl-CoA synthase 1) and Scarb1 (scavenger receptor class B member 1), inhibiting cholesterol synthesis. miR-223 was also found to have an indirect effect on $\mathrm{ABCA} 1$ activity via a repression of $\mathrm{Sp} 3$ 
transcription factor. The expression of miR-223 was itself found to be linked with cholesterol levels, with decreased miR-223 transcription during cholesterol depletion, providing a case for its potential use in diagnostics as a circulating biomarker for altered cholesterol homeostasis. Cholesterol efflux, which has been regarded as an important cholesterol regulatory mechanism, has been shown in other studies to be controlled by miRNAs. miR-33a and miR-33b, located within the intron of the sterol regulatory element-binding protein genes $S R E B F 2$ and $S R E B F 1$, coding for major transcription factors controlling cholesterol and fatty acid metabolism. These are co-transcribed in cholesterol depleted conditions [13]. miR-33a is expressed in human macrophages and hepatocytes and regulates cholesterol efflux and HDL synthesis via gene repression of cholesterol transporter proteins including ABCA1 [14]. ABCA1 (ATP-binding cassette transporter 1), which contains putative binding sites for miR-33a and miR-33b in its 3' UTR, mediates efflux of free cholesterol which then forms HDL and is of particular importance in lipid-laden macrophages (foam cells) for removal of excess cholesterol out of the cell by reverse cholesterol transport. Regulation of ABCA1targeting miRNAs holds great therapeutic potential in the treatment of atherosclerosis, via cholesterol homeostasis as small changes in their expression influences ABCA1 activity, in turn leading to large changes in HDL-cholesterol levels $[15,16]$

While our understanding of the roles of miRNA regulation in lipid metabolism rapidly grows, the practical use of this knowledge in the clinical application of treatments such as anti-miRs and miRNA-mimics for lipid disorders will experience the many challenges RNAi-based therapeutics face, including their need for targeted delivery and the avoidance of immune response activation [10].

\section{Acknowledgements}

None.

\section{Conflicts of interest}

The authors have no conflicts of interest to declare. 


\section{REFERENCES AND RECOMMENDED READING}

[1] Wilczynska A, Bushell M. The complexity of miRNA-mediated repression. Cell Death Differ 2015; 22:22-33.

[2] Griffiths-Jones S, Grocock RJ, van Dongen S et al. miRBase: microRNA sequences, targets and gene nomenclature. Nucleic Acids Res 2006; 34:D140-144.

[3]- Peterson SM, Thompson JA, Ufkin ML et al. Common features of microRNA target prediction tools. Frontiers in genetics 2014; 5:23.

[4] Bartel DP. MicroRNAs: target recognition and regulatory functions. Cell 2009; 136:215-233.

[5] Friedman RC, Farh KK, Burge CB, Bartel DP. Most mammalian mRNAs are conserved targets of microRNAs. Genome Res 2009; 19:92-105.

[6] Ha TY. MicroRNAs in Human Diseases: From Cancer to Cardiovascular Disease. Immune Netw 2011; 11:135-154.

[7] Iguchi H, Kosaka N, Ochiya T. Secretory microRNAs as a versatile communication tool. Commun Integr Biol 2010; 3:478-481.

[8] Russo F, Di Bella S, Nigita G et al. miRandola: extracellular circulating microRNAs database. PLoS One 2012; 7:e47786.

[9] Scholer N, Langer C, Dohner H et al. Serum microRNAs as a novel class of biomarkers: a comprehensive review of the literature. Exp Hematol 2010; 38:11261130 .

[10] van Rooij E, Kauppinen S. Development of microRNA therapeutics is coming of age. EMBO Mol Med 2014; 6:851-864.

[11] He PP, Ouyang XP, Tang YY et al. MicroRNA-590 attenuates lipid accumulation and pro-inflammatory cytokine secretion by targeting lipoprotein lipase gene in human THP-1 macrophages. Biochimie 2014; 106:81-90.

[12]-" Vickers KC, Landstreet SR, Levin MG et al. MicroRNA-223 coordinates cholesterol homeostasis. Proc Natl Acad Sci U S A 2014; 111:14518-14523.

[13] Davalos A, Goedeke L, Smibert P et al. miR-33a/b contribute to the regulation of fatty acid metabolism and insulin signaling. Proc Natl Acad Sci U S A 2011; 108:9232-9237.

[14] Moore KJ, Rayner KJ, Suarez Y, Fernandez-Hernando C. The role of microRNAs in cholesterol efflux and hepatic lipid metabolism. Annu Rev Nutr 2011; 31:49-63.

[15] Ramirez CM, Davalos A, Goedeke L et al. MicroRNA-758 regulates cholesterol efflux through posttranscriptional repression of ATP-binding cassette transporter A1. Arterioscler Thromb Vasc Biol 2011; 31:2707-2714.

[16] Rayner KJ, Sheedy FJ, Esau CC et al. Antagonism of miR-33 in mice promotes reverse cholesterol transport and regression of atherosclerosis. J Clin Invest 2011; 121:2921-2931.

\section{Annotated bibliography:}

3 - Peterson SM, Thompson JA, Ufkin ML et al. Common features of microRNA target prediction tools. Frontiers in genetics 2014; 5:23. This is an important review of the available computational analysis tools available for predicting miRNA sequences that have potential regulatory cross-disciplinary targets. 
11 - He PP, Ouyang XP, Tang YY et al. MicroRNA-590 attenuates lipid accumulation and pro-inflammatory cytokine secretion by targeting lipoprotein lipase gene in human THP-1 macrophages. Biochimie 2014; 106:81-90.

In this study the effects of miRNA-590 (thought to have protective actions in cardiovascular disease with an unknown target mechanism) were evaluated on the human monocytic cell line THP-1. The authors showed that miRNA-590 inhibited lipoprotein lipase activity and the production of proinflammatory cytokines from these cells. This also coincided with reduced lipid accumulation indicating a possible anti-atherosclerotic mechanism.

12 - Vickers KC, Landstreet SR, Levin MG et al. MicroRNA-223 coordinates cholesterol homeostasis. Proc Natl Acad Sci U S A 2014; 111:14518-14523. This study focussed on miRNA-223 which has been linked to inflammatory responses. The authors showed that miRNA-223 caused repression of the sterol enzymes 3-hydroxy-3-methylglutaryl-CoA synthase 1 and methylsterol monooxygenase 1 thereby inhibiting cholesterol synthesis. It was also able to indirectly increase cholesterol efflux.

\section{Other annotated bibliography from recent papers}

A- van Rooij E, Kauppinen S. Development of microRNA therapeutics is coming of age. EMBO Mol Med 2014; 6:851-64

This paper assesses the potential of miRNAs for the treatment of a wide variety of diseases ranging from chronic viral infection (hepatitis $\mathrm{C}$ virus) and cardiovascular disease to cancer. Different strategies for the modulation of miRNA activity are discussed such as miRNA mimics and antimiR oligonucleotides.

B- Daniel JM, Penzkofer D, Teske R et al. Inhibition of miR-92a improves reendothelialization and prevents neointima formation following vascular injury. Cardiovasc Res 2014; 103:564-72

This study investigated the role of miRNA-92a in vascular injury. The authors observed that miRNA-92a was upregulated following physically-induced damage to femoral arteries from mice. They were also able to show that, using antisense locked nucleic acid to miRNA-92a, suppression of intimal lesions occurred as a result of accelerated re-endothelialisation uncovering a potential approach to the treatment of vascular injury.

C- [1] Kopke S, Buhrke T, Lampen A. miRNA Expression in Human Intestinal Caco-2 Cells is Comparably Regulated by cis- and trans-Fatty Acids. Lipids 2015; 50:227-39.

This study set out to evaluate whether there were any differences in miRNA expression in response to either trans- or cis-fatty acids in cancer cells. The authors investigated the expression of 84 cancer-related miRNAs in Caco- 2 
cells (colon cancer-derived). They concluded that there was no bias toward either trans- or cis-fatty acids in 66 miRNAs that were deregulated by fatty acids. 